\title{
Exact solutions for an oscillator with anti-symmetric quadratic nonlinearity
}
A. Beléndez, ${ }^{1,2}$
F. J. Martínez, ${ }^{2}$ T. Beléndez, ${ }^{1,2}$
C. Pascual, ${ }^{1,2}$
M. L. Alvarez, ${ }^{1,2}$
E. Gimeno ${ }^{1,2}$ and
E. Arribas ${ }^{3}$

(1) Instituto Universitario de Física Aplicada a las Ciencias y las Tecnologías.

Universidad de Alicante. Apartado 99. E-03080 Alicante. SPAIN

(2) Departamento de Física, Ingeniería de Sistemas y Teoría de la Señal.

Universidad de Alicante. Apartado 99. E-03080 Alicante. SPAIN

(3) Departamento de Física Aplicada. Universidad de Castilla-La Mancha.

Avda. España s/n, E-02071 Albacete. SPAIN

\begin{abstract}
Closed-form exact solutions for an oscillator with anti-symmetric quadratic nonlinearity are derived from the first integral of the nonlinear differential equation governing the behaviour of this oscillator. The mathematical model is an ordinary second order differential equation in which the sign of the quadratic nonlinear term changes. Two parameters characterize this oscillator: the coefficient of the linear term and the coefficient of the quadratic term. Not only the common case in which both coefficients are positive but also all possible combinations of positive and negative signs of these coefficients which provide periodic motions are considered, giving rise to four different cases. Three different periods and solutions are obtained, since the same result is valid in two of these cases. An interesting feature is that oscillatory motions whose equilibrium points are not at $x=0$ are also considered. The periods are given in terms of an incomplete or complete elliptic integral of the first kind, and the exact solutions are expressed as functions including Jacobi elliptic cosine or sine functions.
\end{abstract}

Keywords: Dynamical systems; Nonlinear oscillators; Conservative systems; Elliptic integrals; Jacobi elliptic functions

PACS: 05.45.-a, 02.30.Hq, 05.10.-a 


\section{Introduction}

Mathematical models based on nonlinear oscillators have been widely used, not only in mechanics but also in other branches of physics, mathematics or engineering [1-4]. In conservative nonlinear oscillators the restoring force is not dependent on time, the total energy is constant [2,3] and any oscillation is stationary. The solutions for this type of oscillators are periodic and range over a continuous interval of initial values [5]. In this paper we obtain closed-form exact expressions for the period and solution of an anti-symmetric quadratic nonlinear oscillator with linear and nonlinear terms, modelled by the following second-order differential equation

$$
\frac{\mathrm{d}^{2} x}{\mathrm{~d} t^{2}}+a_{1} x+a_{2} \operatorname{sgn}(x) x^{2}=0
$$

where $a_{1}$ and $a_{2}$ are the coefficients of the linear and nonlinear terms, respectively. Cveticanin analysed the vibrations of this oscillator when $a_{1}>0$ [6] and made a qualitative analysis of the differential equation in (1) for both mass-hard and mass-soft spring systems. She also derived the exact solution to the anti-symmetric quadratic equation when $a_{1}>0$ using Jacobi elliptic functions [6]. Elías Zúñiga obtained the exact solution to the quadratic mixed-parity Helmholtz-Duffing oscillator [7]; Jin-wen Zhu derived the exact solution to a damped quadratic non-linear oscillator [8], and Marinca and Herişanu obtained explicit and exact solutions to cubic Duffing and double-well Duffing equations [9]. However, none of these authors solved the nonlinear differential equation but assumed that its exact solution is given by an equation which includes the dn Jacobian elliptic function and five unknown parameters that need to be determined. When $a_{1}=0$ and $a_{2}>0$, Eq. (1) corresponds to a truly nonlinear oscillator [10] for which exact expressions for the period and solution have already been obtained [11]. However, as far as we know, for the general situation described by Eq. (1), only analytical approximate expressions for the exact period and exact solution have so far been obtained. Mickens and Mixon [12] obtained accurate analytical approximate solutions to an anti-symmetric quadratic non-linear oscillator using the generalized harmonic balance method, as did Chen et al [13] using the elliptic perturbation method. Beléndez et al approximately solved this nonlinear oscillator when $a_{1}=0$ using a modified He's homotopy perturbation method [14] as well as a novel rational harmonic balance approach [15]. Recently, Cveticanin et al [16] obtained solutions to oscillators with symmetric and 
asymmetric quadratic nonlinearity in the form of Jacobi elliptic functions. The case in which coefficients $a_{1}$ and $a_{2}$ are both positive is the most commonly analysed. However, we obtained exact approximate solutions not only when both coefficients are positive but also for all possible combinations of positive and negative values of these coefficients which provide periodic motions. In this paper we obtain exact expressions for the period and solution of an anti-symmetric quadratic nonlinear oscillator modelled by Eq. (1). Unlike the procedure followed by other authors [6-9,16], instead of assuming an expression for the solution we solve the nonlinear differential equation exactly. The various combinations of positive and negative signs of these coefficients give rise to four different cases. In three of these ((a) $a_{1} \geq 0, a_{2}>0$, and $x_{0}>0$, (b) $a_{1}<0, a_{2}>0$ and $x_{0}>-3 a_{1} / 2 a_{2}$, and (c) $a_{1}>0, a_{2}<$ 0 and $\left.0<x_{0}<-a_{1} / a_{2}\right)$ the system oscillates around the equilibrium position $x=0$ with $x \in\left[-x_{0}, x_{0}\right]$, where $x_{0}>0$ is the oscillation amplitude. However, there is still one more case ((d) $a_{1}>0, a_{2}<0$, and $0>x_{1}>-3 a_{1} / 2 a_{2}$ or $\left.3 a_{1} / 2 a_{2}<x_{0}<0\right)$ in which the system does not oscillate around the position $x=0$ where $x \in\left[-x_{0}, x_{0}\right]$, but around the equilibrium position $x=-a_{1} / a_{2}$ where $x \in\left[x_{1}, x_{0}\right]$ (when $0>x_{0}>-3 a_{1} / 2 a_{2}$ ) or $x=a_{1} / a_{2}$ where $x \in\left[x_{1}, x_{0}\right]$ (when $3 a_{1} / 2 a_{2}<x_{0}<0$ ). Three different sets of closed-form expressions for the exact period and solution were obtained. Some examples are analysed and plots including periods, solutions or phase-diagrams are presented and discussed.

\section{Solution when $a_{1} \geq 0, a_{2}>0$ and $x_{0}>0$}

Consider the anti-symmetric quadratic nonlinear oscillator given in Eq. (1) with initial conditions

$$
x(0)=A \quad \frac{\mathrm{d} x}{\mathrm{~d} t}(0)=0
$$

In Eq. (1) we assume that the coefficients of the linear and the nonlinear terms satisfy the inequalities

$$
a_{1} \geq 0 \quad a_{2}>0
$$


This system corresponds to a nonlinear oscillator for which the nonlinear function $f(x)=a_{1} x+a_{2} \operatorname{sgn}(x) x^{2}$ is odd, i.e. $f(-x)=-f(x)$ and satisfies the condition $x f(x)>0$ when $x \in\left[-x_{0}, x_{0}\right], x \neq 0$, where $x_{0}>0$ is the initial amplitude. This system oscillates around the equilibrium position $x=0$ and the period, $T$, and periodic solution, $x$, are dependent on the oscillation amplitude $x_{0}$.

In order to obtain the exact period and periodic solution to Eq. (1), we take into account that this is a conservative system and has the following first integral

$$
\left(\frac{\mathrm{d} x}{\mathrm{~d} t}\right)^{2}+a_{1} x^{2}+\frac{2}{3} a_{2} \operatorname{sgn}(x) x^{3}=a_{1} x_{0}^{2}+\frac{2}{3} a_{2} x_{0}^{3} \geq 0
$$

which can be written as follows

$$
\left(\frac{\mathrm{d} x}{\mathrm{~d} t}\right)^{2}=a_{1}\left(x_{0}^{2}-x^{2}\right)+\frac{2}{3} a_{2}\left(x_{0}^{3}-\operatorname{sgn}(x) x^{3}\right)
$$

From this equation we obtain

$$
\sqrt{\frac{2 a_{2}}{3}} \mathrm{~d} t= \pm \frac{\mathrm{d} x}{\sqrt{\frac{3 a_{1}}{2 a_{2}}\left(x_{0}^{2}-x^{2}\right)+\left(x_{0}^{3}-\operatorname{sgn}(x) x^{3}\right)}}
$$

where the sign $( \pm)$ is chosen taking into account the sign of $\mathrm{d} x / \mathrm{d} t$ in each quadrant.

From Eq. (6) we obtain $t$ as a function of $x$ for the following cases:

(a) Trajectory $1 \rightarrow 2\left(0 \leq t \leq T / 4\right.$ and $\left.x_{0} \geq x \geq 0\right), x$ is positive and $\mathrm{d} x / \mathrm{d} t$ is negative.

(b) Trajectory $2 \rightarrow 3\left(T / 4 \leq t \leq T / 2\right.$ and $\left.0 \geq x \geq-x_{0}\right), x$ is negative and $\mathrm{d} x / \mathrm{d} t$ is negative.

(c) Trajectory $3 \rightarrow 4\left(T / 2 \leq t \leq 3 T / 4\right.$ and $\left.-x_{0} \leq x \leq 0\right), x$ is negative and $\mathrm{d} x / \mathrm{d} t$ is positive.

(d) Trajectory $4 \rightarrow 1\left(3 T / 4 \leq t \leq T\right.$ and $\left.0 \leq x \leq x_{0}\right), x$ is positive and $\mathrm{d} x / \mathrm{d} t$ is positive:

For the first time interval $(0 \leq t \leq T / 4$, trajectory $1 \rightarrow 2)$ the deflection is positive $(x>0)$ and from Eq. (6) it follows that 


$$
\sqrt{\frac{2 a_{2}}{3}} \mathrm{~d} t=-\frac{\mathrm{d} x}{\sqrt{\frac{3 a_{1}}{2 a_{2}}\left(x_{0}^{2}-x^{2}\right)+\left(x_{0}^{3}-x^{3}\right)}}
$$

and after some mathematical operations we obtain

$$
\sqrt{\frac{2 a_{2}}{3}} \int_{0}^{t} \mathrm{~d} t=-\int_{x_{0}}^{x} \frac{\mathrm{d} x}{\sqrt{\left(x_{0}-x\right)\left[x^{2}+\left(x_{0}+\frac{3 a_{1}}{2 a_{2}}\right) x+x_{0}^{2}+\frac{3 a_{1}}{2 a_{2}} x_{0}\right]}}
$$

which can be written as

$$
\sqrt{\frac{2 a_{2}}{3}} t=\int_{x}^{\alpha} \frac{\mathrm{d} x}{\sqrt{(\alpha-x)\left[(x-\sigma)^{2}+\rho^{2}\right]}}
$$

where

$$
\begin{aligned}
& \alpha=x_{0} \\
& \sigma=-\frac{1}{2} x_{0}-\frac{3 a_{1}}{4 a_{2}} \\
& \rho=\sqrt{\frac{3}{4} x_{0}^{2}+\frac{3 a_{1}}{4 a_{2}} x_{0}-\frac{9}{16}\left(\frac{a_{1}}{a_{2}}\right)^{2}}
\end{aligned}
$$

The value of the integral in Eq. (9) is given in GR's book $a \geq x \geq b>c\left[17,6^{\text {th }}\right.$ edition, Formula 3.138, Integral 8, page 260]; however, we found an error in the calculation (an inverse cotangent function is included instead of an arctangent function). Consequently, we solved the integral ourselves (see Appendix A) and obtained the following result (Eq. (A11)):

$$
\int_{x}^{\alpha} \frac{\mathrm{d} u}{\sqrt{(\alpha-x)\left[(x-\sigma)^{2}+\rho^{2}\right]}}=\frac{1}{\sqrt{p}} F\left(2 \arctan \sqrt{\frac{\alpha-x}{p}}, \frac{p-\sigma+\alpha}{2 p}\right)
$$

where

$$
p=\sqrt{(\sigma-\alpha)^{2}+\rho^{2}}
$$


and $F(\phi, m)$ is the incomplete elliptic integral of the first kind defined as follows [15]

$$
F(\phi, m)=\int_{0}^{\phi} \frac{\mathrm{d} \theta}{\sqrt{1-m \sin ^{2} \theta}}
$$

Therefore, from Eqs. (10)-(15) it follows that

$$
\begin{gathered}
p=\sqrt{\frac{3 a_{1}}{a_{2}} x_{0}+3 x_{0}^{2}} \\
m=k^{2}=\frac{p-\sigma+\alpha}{2 p}=\frac{1}{2}+\frac{3 a_{1}+6 a_{2} x_{0}}{8 \sqrt{3 a_{2}\left(a_{1} x_{0}+a_{2} x_{0}^{2}\right)}}
\end{gathered}
$$

where $m$ is the parameter and $k$ is the elliptic modulus. The inverse function of $F(\phi, m)$ is given by the Jacobi amplitude $\phi[18,19]$

$$
F^{-1}(u, m)=\phi=\operatorname{am}(u, m)
$$

whose cosine is the Jacobi cosine function, cn [19]

$$
\cos \phi=\cos (\operatorname{am}(u, m))=\operatorname{cn}(u, m)
$$

In order to introduce an "arccos" function in Eq. (14) we take into account that

$$
2 \arctan z=\phi
$$

where

$$
z=\sqrt{\frac{\alpha-x}{p}}
$$

From Eq. (20) we obtain

$$
\tan \frac{\phi}{2}=z
$$

Taking into account the trigonometric formula

$$
\tan \frac{\phi}{2}= \pm \sqrt{\frac{1-\cos \phi}{1+\cos \phi}}
$$

it follows that 


$$
\cos \phi=\frac{1-z^{2}}{1+z^{2}}
$$

which allows Eq. (14) to be written as

$$
\int_{x}^{\alpha} \frac{\mathrm{d} x}{\sqrt{(\alpha-x)\left[(x-\sigma)^{2}+\rho^{2}\right]}}=\frac{1}{\sqrt{p}} F\left(\arccos \left(\frac{p-\alpha+x}{p+\alpha-x}\right), \frac{p-\sigma+\alpha}{2 p}\right)
$$

From Eqs. (9), (19) and (25), it follows that

$$
\frac{p-\alpha+x}{p+\alpha-x}=\operatorname{cn}\left(\sqrt{\frac{2 a_{2} p}{3}} t, \frac{p-\sigma+\alpha}{2 p}\right)
$$

Substituting Eqs. (10), (11), (12), (16) and (17) into Eq. (26) gives

$$
\left.x_{a}(t)=x_{0}-\sqrt{\frac{3 a_{1}}{a_{2}} x_{0}+3 x_{0}^{2}}\left(\frac{1-\operatorname{cn}\left(\left(\frac{4}{3} a_{2}\left(a_{1} x_{0}+a_{2} x_{0}^{2}\right)\right)^{1 / 4} t, m\right)}{1+\operatorname{cn}\left(\left(\frac{4}{3} a_{2}\left(a_{1} x_{0}+a_{2} x_{0}^{2}\right)\right)^{1 / 4} t, m\right)}\right)\right)
$$

which is valid for trajectory $1 \rightarrow 2$ and $m$ is given in Eq. (17).

The period of oscillation is four times the time taken by the oscillator to go from $x=0$ to $x=$ $x_{0}$. This results in

$$
T=4 \sqrt{\frac{3}{2 a_{2}}} \int_{0}^{\alpha} \frac{\mathrm{d} x}{\sqrt{(\alpha-x)\left[(x-\sigma)^{2}+\rho^{2}\right]}}=4 \sqrt{\frac{3}{2 p a_{2}}} F\left(2 \arctan \sqrt{\frac{\alpha}{p}}, \frac{p-\sigma+\alpha}{2 p}\right)
$$

and substituting Eqs. (10), (11), (16) and (17) into Eq. (28) gives

$$
T=\frac{2 \sqrt{6}}{\left(3 a_{1} a_{2} x_{0}+3 a_{2}^{2} x_{0}^{2}\right)^{1 / 4}} F\left(2 \arctan \left(\frac{a_{2} x_{0}}{3 a_{1}+3 a_{2} x_{0}}\right)^{1 / 4}, \frac{1}{2}+\frac{3 a_{1}+6 a_{2} x_{0}}{8 \sqrt{3 a_{2}\left(a_{1} x_{0}+3 a_{2} x_{0}^{2}\right)}}\right)
$$

Taking into account Eq. (25) the exact period can also be written as

$$
T=\frac{2 \sqrt{6}}{\left(3 a_{1} a_{2} x_{0}+3 a_{2}^{2} x_{0}^{2}\right)^{1 / 4}} F\left(\arccos \left(\frac{\sqrt{3 a_{1} x_{0}+3 a_{2} x_{0}^{2}}-\sqrt{a_{2}} x_{0}}{\sqrt{3 a_{1} x_{0}+3 a_{2} x_{0}^{2}}+\sqrt{a_{2}} x_{0}}\right), \frac{1}{2}+\frac{3 a_{1}+6 a_{2} x_{0}}{8 \sqrt{3 a_{2}\left(a_{1} x_{0}+3 a_{2} x_{0}^{2}\right)}}\right)
$$

which can be written as follows 


$$
T=\frac{F\left(\phi_{0}, m\right)}{\Omega}
$$

where

$$
\begin{gathered}
\phi_{0}\left(a_{1}, a_{2}, x_{0}\right)=\arccos \left(\frac{\sqrt{3 a_{1} x_{0}+3 a_{2} x_{0}^{2}}-\sqrt{a_{2}} x_{0}}{\sqrt{3 a_{1} x_{0}+3 a_{2} x_{0}^{2}}+\sqrt{a_{2}} x_{0}}\right) \\
\Omega\left(a_{1}, a_{2}, x_{0}\right)=\frac{1}{2 \sqrt{6}}\left(3 a_{1} a_{2} x_{0}+3 a_{2}^{2} x_{0}^{2}\right)^{1 / 4}
\end{gathered}
$$

and $m$ is given in Eq. (17).

In the next time-motion interval $(T / 4 \leq t \leq T / 2$, trajectory $2 \rightarrow 3)$ the deflection is negative, $x$ $<0$ and from Eq. (6) it follows that

$$
\sqrt{\frac{2 a_{2}}{3}} \int_{T / 4}^{t} \mathrm{~d} t=-\int_{0}^{x} \frac{\mathrm{d} x}{\sqrt{\frac{3 a_{1}}{2 a_{2}}\left(x_{0}^{2}-x^{2}\right)+\left(x_{0}^{3}+x^{3}\right)}}
$$

where $T$ is the period of oscillation given in Eq. (29). Let $x=-y$, then Eq. (34) becomes

$$
\sqrt{\frac{2 a_{2}}{3}}\left(t-\frac{T}{4}\right)=\int_{0}^{-x} \frac{\mathrm{d} y}{\sqrt{\frac{3 a_{1}}{2 a_{2}}\left(x_{0}^{2}-y^{2}\right)+\left(x_{0}^{3}-y^{3}\right)}}
$$

which can be written as follows

$$
\sqrt{\frac{2 a_{2}}{3}}\left(t-\frac{T}{4}\right)=\int_{0}^{x_{0}} \frac{\mathrm{d} y}{\sqrt{\frac{3 a_{1}}{2 a_{2}}\left(x_{0}^{2}-y^{2}\right)+\left(x_{0}^{3}-y^{3}\right)}}-\int_{-x}^{x_{0}} \frac{\mathrm{d} y}{\sqrt{\frac{3 a_{1}}{2 a_{2}}\left(x_{0}^{2}-y^{2}\right)+\left(x_{0}^{3}-y^{3}\right)}}
$$

The values of the two integrals on the right-hand side of Eq. (36) are

$$
\int_{0}^{x_{0}} \frac{\mathrm{d} y}{\sqrt{\frac{3 a_{1}}{2 a_{2}}\left(x_{0}^{2}-y^{2}\right)+\left(x_{0}^{3}-y^{3}\right)}}=\sqrt{\frac{2 a_{2}}{3}} \frac{T}{4}
$$




$$
\int_{-x}^{x_{0}} \frac{\mathrm{d} y}{\sqrt{\frac{3 a_{1}}{2 a_{2}}\left(x_{0}^{2}-y^{2}\right)+\left(x_{0}^{3}-y^{3}\right)}}=\frac{1}{\sqrt{p}} F\left(\arccos \left(\frac{p-\alpha-x}{p+\alpha+x}\right), \frac{p-\sigma+\alpha}{2 p}\right)
$$

where Eqs. (25) and (29) have been taken into account. Substituting Eqs. (37) and (38) into Eq. (34) gives

$$
\frac{p-\alpha-x}{p+\alpha+x}=\operatorname{cn}\left(\sqrt{\frac{2 a_{2} p}{3}}\left(\frac{T}{2}-t\right), \frac{p-\sigma+\alpha}{2 p}\right)
$$

After some simplifications, the solution for trajectory $2 \rightarrow 1$ can be written as follows

$$
\left.x_{b}(t)=-x_{0}+\sqrt{\frac{3 a_{1}}{a_{2}} x_{0}+3 x_{0}^{2}}\left(\frac{1-\operatorname{cn}\left(\left(\frac{4}{3} a_{2}\left(a_{1} x_{0}+a_{2} x_{0}^{2}\right)\right)^{1 / 4}\left(t-\frac{T}{2}\right), m\right)}{1+\operatorname{cn}\left(\left(\frac{4}{3} a_{2}\left(a_{1} x_{0}+a_{2} x_{0}^{2}\right)\right)^{1 / 4}\left(t-\frac{T}{2}\right), m\right)}\right)\right)
$$

where we take into account that $\operatorname{cn}\left(z_{1}-z_{2} ; m\right)=\operatorname{cn}\left(z_{2}-z_{1} ; m\right)$ [16]. For trajectory $3 \rightarrow 4(T / 2$ $\leq t \leq 3 T / 4)$ the deflection is also negative $(x<0)$ and it can easily be verified that the same solution as that given in Eq. (40) is obtained.

Finally, for the last time interval $(3 T / 4 \leq t \leq T$, trajectory $4 \rightarrow 1)$ the deflection is positive and from Eq. (6) it follows that

$$
\sqrt{\frac{2 a_{2}}{3}} \int_{3 T / 4}^{t} \mathrm{~d} t=\int_{0}^{x} \frac{\mathrm{d} x}{\sqrt{\left(x_{0}-x\right)\left[x^{2}+\left(x_{0}+\frac{3 a_{1}}{2 a_{2}}\right) x+x_{0}^{2}+\frac{3 a_{1}}{2 a_{2}} x_{0}\right]}}
$$

which can be written as follows

$$
\sqrt{\frac{2 a_{2}}{3}}\left(t-\frac{3 T}{4}\right)=\int_{0}^{x_{0}} \frac{\mathrm{d} x}{\sqrt{\frac{3 a_{1}}{2 a_{2}}\left(x_{0}^{2}-x^{2}\right)+\left(x_{0}^{3}-x^{3}\right)}}-\int_{x}^{x_{0}} \frac{\mathrm{d} x}{\sqrt{\frac{3 a_{1}}{2 a_{2}}\left(x_{0}^{2}-x^{2}\right)+\left(x_{0}^{3}-x^{3}\right)}}
$$

After simplifying the solution for $3 \rightarrow 4$ may be written as follows 


$$
x_{c}(t)=x_{0}-\sqrt{\frac{3 a_{1}}{a_{2}} x_{0}+3 x_{0}^{2}}\left(\frac{1-\mathrm{cn}\left(\left(\frac{4}{3} a_{2}\left(a_{1} x_{0}+a_{2} x_{0}^{2}\right)\right)^{1 / 4}(t-T), m\right)}{1+\operatorname{cn}\left(\left(\frac{4}{3} a_{2}\left(a_{1} x_{0}+a_{2} x_{0}^{2}\right)\right)^{1 / 4}(t-T), m\right)}\right)
$$

Then, the exact solution to Eq. (1) for the first period $(0 \leq t \leq T)$ can be written as the following piecewise function

$$
x(t)=\left\{\begin{array}{cc}
x_{a}(t), & 0 \leq t \leq \frac{T}{4} \\
-x_{a}\left(t-\frac{T}{2}\right), & \frac{T}{4} \leq t \leq \frac{3 T}{4} \\
x_{a}(t-T), & \frac{3 T}{4} \leq t \leq T
\end{array}\right.
$$

where $x(t)$ is a continuous function over the entire domain and $x_{a}(t), x_{b}(\mathrm{t})$ and $x_{c}(t)$ are given in Eqs. (27), (40) and (43), respectively.

In summary, when $a_{1} \geq 0$ and $a_{2}>0$, and for all values of the initial amplitude $\left(x_{0}>0\right)$, the exact period is given by Eq. (29) and the exact solution is a piece-wise continuous timedependent function composed of three parts defined separately over two quarter-period and one half-period intervals as can be seen in Eq. (44).

We can obtain the period when $a_{1}=0$ and $a_{2}=1$, which corresponds to a truly nonlinear oscillator [10]

$$
\frac{\mathrm{d}^{2} x}{\mathrm{~d} t^{2}}+\operatorname{sgn}(x) x^{2}=0
$$

From Eq. (29) the period of this truly nonlinear oscillator is

$$
T=\frac{3^{1 / 4} \sqrt{8}}{\sqrt{x_{0}}} F\left(2 \arctan \left(\frac{1}{3^{1 / 4}}\right), \frac{2+\sqrt{3}}{4}\right)=\frac{3^{1 / 4} \sqrt{8}}{\sqrt{x_{0}}} F\left(\arccos \left(\frac{\sqrt{3}-1}{\sqrt{3}-1}\right), \frac{2+\sqrt{3}}{4}\right) \approx \frac{6.86926}{\sqrt{x_{0}}}
$$

which coincides with the value given in [7]

$$
T=\sqrt{\frac{8 \pi}{3 x_{0}}} \frac{\Gamma(1 / 3)}{\Gamma(5 / 6)} \approx \frac{6.86926}{\sqrt{x_{0}}}
$$




\section{Solution when $a_{1}<0, a_{2}>0$ and $x_{0}>-3 a_{1} / 2 a_{2}$}

We assumed that $a_{1} \geq 0$ and $a_{2}>0$ (Eq. (3)) in order to obtain the exact period and exact solution given in Eqs. (27), (30), (40) and (43) which are valid for all values of the initial amplitude $\left(x_{0}>0\right)$. However, under certain conditions these equations are also valid even though $a_{1}$ and $a_{2}$ are not both positive. A dynamical study of the nonlinear differential equation given in Eq. (1) showed that its motion is periodic with a centre at $(0,0)$ in the following situations:

$$
\left.\begin{array}{llc}
\text { (a) } a_{1} \geq 0, & a_{2}>0, & x_{0}>0 \\
\text { (b) } a_{1}<0, & a_{2}>0, & x_{0}>\frac{3\left|a_{1}\right|}{2 a_{2}} \\
\text { (c) } a_{1}>0, & a_{2}<0, & 0<x_{0}<\frac{a_{1}}{\left|a_{2}\right|}
\end{array}\right\}
$$

The phase plots in Figure 1 illustrate three examples of these situations. Figure 1 (a) shows the behaviour of the oscillator when $a_{1}=1, a_{2}=1$ and $x_{0}=1$ ( $x_{0}$ must be $>0$, (a)). Figure 1 (b) shows its behaviour when $a_{1}=-1, a_{2}=1$ and $x_{0}=1.6\left(x_{0}\right.$ must be $>3 / 2$, (b)), whereas Figure 1 (c) shows the phase plot when $a_{1}=1, a_{2}=-1$ and $x_{0}=0.9$ ( $x_{0}$ must be $<1$, (c)). As can be seen in these figures, the system oscillates around the equilibrium position $x=0$ and the periodic solution $x$ satisfies the condition $x \in\left[-x_{0}, x_{0}\right]$, where $x_{0}>0$ is the oscillation amplitude. Eqs. (27), (30), (40) and (43) can be used to obtain the period and solution in cases (a) and (b), i.e., they are valid for (a) $a_{1} \geq 0, a_{2}>0$ and $x_{0}>0$, and (b) $a_{1}<0, a_{2}>0$ and $x_{0}>\frac{3\left|a_{1}\right|}{2 a_{2}}$. However, in case (c) in Eq. (48) it is necessary to obtain the equation for the period again since if $a_{2}<0$, the root $\sqrt{2 a_{2} / 3}$ is not a real number and so Eq. (6) can not be used. 

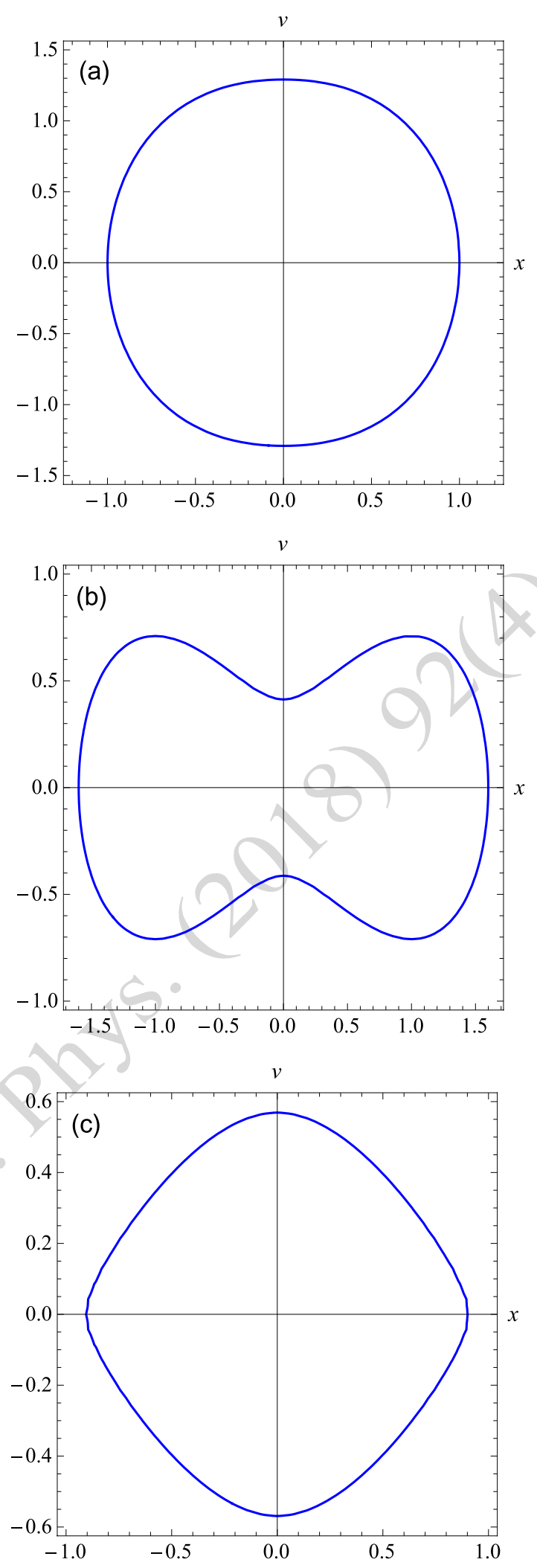

Figure 1.- Phase plot for (a) $a_{1}=1$ and $a_{2}=1$ with $x_{0}=1$ ( $x_{0}$ must be $>0$, (a)), (b) $a_{1}=-1$ and $a_{2}=$ 1 with $x_{0}=1.6$ ( $x_{0}$ must be $>3 / 2$, (b)), and (c) $a_{1}=1$ and $a_{2}=-1$ with $x_{0}=0.9$ ( $x_{0}$ must be $<1,(\mathrm{c}))$. 


\section{Solution when $a_{1}>0, a_{2}<0$ and $0<x_{0}<-a_{1} / a_{2}$}

In order to obtain the exact solution when $a_{1}>0, a_{2}<0$ and $0<x_{0}<a_{1} /\left|a_{2}\right|$ (Eq. (48c)) in which case the system oscillates around the equilibrium position $x=0$ and the periodic solution $x$ satisfies the condition $x \in\left[-x_{0}, x_{0}\right]$, we proceed as follows. From Eq. (5) and considering that $x>0$, we obtain $t$ as a function of $x$ as follows

$$
\sqrt{\frac{-2 a_{2}}{3}} \mathrm{~d} t= \pm \frac{\mathrm{d} x}{\sqrt{-\frac{3 a_{1}}{2 a_{2}}\left(x_{0}^{2}-x^{2}\right)-\left(x_{0}^{3}-x^{3}\right)}}
$$

Taking into account that $a_{1}<0$ and $a_{2}<0$, Eq. (49) can be written as

$$
\sqrt{\frac{-2 a_{2}}{3}} \int_{0}^{t} \mathrm{~d} t= \pm \int_{x}^{b} \frac{\mathrm{d} x}{\sqrt{(a-x)(b-x)(x-c)}}
$$

where constants $a, b$ and $c$ satisfy the condition that $a>b \geq x>c$ and are given as follows

$$
\begin{aligned}
& a=\frac{1}{4 a_{2}}\left(-3 a_{1}-2 a_{2} x_{0}-\sqrt{3} \sqrt{3 a_{1}^{2}-4 a_{1} a_{2} x_{0}-4 a_{2}^{2} x_{0}^{2}}\right) \\
& b=x_{0} \\
& c=\frac{1}{4 a_{2}}\left(-3 a_{1}-2 a_{2} x_{0}+\sqrt{3} \sqrt{3 a_{1}^{2}-4 a_{1} a_{2} x_{0}-4 a_{2}^{2} x_{0}^{2}}\right)
\end{aligned}
$$

The value of the integral in Eq. (50) when $a \geq x \geq b>c$ is $\left[17,6^{\text {th }}\right.$ edition, Formula 3.131, Integral 4, page 250]

$$
\int_{x}^{b} \frac{d x}{\sqrt{(a-x)(x-b)(x-c)}}=\frac{2}{\sqrt{a-c}} F(\delta, q)
$$

$F(\phi, m)$ is the incomplete elliptic integral of the first kind defined in Eq. (15) and

$$
\begin{gathered}
\delta=\arcsin \sqrt{\frac{(a-c)(b-x)}{(b-c)(a-x)}} \\
q=\frac{b-c}{a-c}
\end{gathered}
$$


Substitution of these results into Eq. (50) gives the following equation for $t$ as a function of $x$

$$
t=2 \sqrt{\frac{3}{2 a_{2}(c-a)}} F\left(\arcsin \sqrt{\frac{(a-c)\left(x_{0}-x\right)}{\left(x_{0}-c\right)(a-x)}}, \frac{x_{0}-c}{a-c}\right)
$$

The period of oscillation is four times the time taken by the oscillator to go from $x=x_{0}$ to $x=$ 0 . Therefore

$$
T=8 \sqrt{\frac{3}{2 a_{2}(c-a)}} F\left(\arcsin \sqrt{\frac{(a-c) x_{0}}{\left(x_{0}-c\right) a}}, \frac{x_{0}-c}{a-c}\right)
$$

which finally can be written as follows

$$
T=8 \sqrt{\frac{3}{\Phi}} F\left(\arcsin \sqrt{\frac{2 \Phi}{9 a_{1}+6 a_{2} x_{0}+\Phi}}, \frac{\Phi-3 a_{1}-6 a_{2} x_{0}}{2 \Phi}\right)
$$

where $\Phi$, which is a function of $a_{1}, a_{2}$ and $x_{0}$, is defined as follows

$$
\Phi\left(a_{1}, a_{2}, x_{0}\right)=\sqrt{3} \sqrt{3 a_{1}^{2}-4 a_{1} a_{2} x_{0}-4 a_{2}^{2} x_{0}^{2}}
$$

From Eq. (57) it follows that if $0 \leq t \leq T / 4$ then

$$
F\left(\arcsin \sqrt{\frac{(a-c)(A-x)}{(A-c)(a-x)}}, \frac{A-c}{a-c}\right)=\frac{1}{2} \sqrt{\frac{2 a_{2}(c-a)}{3}} t
$$

The inverse function of the incomplete elliptical function of the first kind in Eq. (61) is the Jacobi sine function sn. Therefore

$$
\sqrt{\frac{(a-c)\left(x_{0}-x\right)}{\left(x_{0}-c\right)(a-x)}}=\operatorname{sn}\left(\frac{1}{2} \sqrt{\frac{2 a_{2}(c-a)}{3}} t, \frac{x_{0}-c}{a-c}\right)
$$

and after some mathematical operations, when $0 \leq t \leq T / 4$ we finally obtain the following solution

$$
x_{a}(t)=x_{0}\left[\frac{1-\frac{9 a_{1}+6 a_{2} x_{0}+\Phi}{2 \Phi} \operatorname{sn}^{2}\left(\frac{1}{2} \sqrt{\frac{\Phi}{3}} t, \frac{\Phi-3 a_{1}-6 a_{2} x_{0}}{2 \Phi}\right)}{1+\frac{9 a_{1}+6 a_{2} x_{0}+\Phi}{2 \Phi} \operatorname{sn}^{2}\left(\frac{1}{2} \sqrt{\frac{\Phi}{3}} t, \frac{\Phi-3 a_{1}-6 a_{2} x_{0}}{2 \Phi}\right)}\right]
$$


where $\Phi$ is given in Eq. (60).

In a similar way it is possible to obtain the following equation for the exact solution when $T / 4 \leq t \leq 3 T / 4$

$$
x_{b}(t)=-x_{0}\left[\frac{1-\frac{9 a_{1}+6 a_{2} x_{0}+\Phi}{2 \Phi} \operatorname{sn}^{2}\left(\frac{1}{2} \sqrt{\frac{\Phi}{3}}\left(t-\frac{T}{2}\right), \frac{\Phi-3 a_{1}-6 a_{2} x_{0}}{2 \Phi}\right)}{1+\frac{9 a_{1}+6 a_{2} x_{0}+\Phi}{2 \Phi} \operatorname{sn}^{2}\left(\frac{1}{2} \sqrt{\frac{\Phi}{3}}\left(t-\frac{T}{2}\right), \frac{\Phi-3 a_{1}-6 a_{2} x_{0}}{2 \Phi}\right)}\right]
$$

and finally when $3 T / 4 \leq t \leq T$ the exact solution is given by the following equation

$$
x_{c}(t)=x_{0}\left[\frac{1-\frac{9 a_{1}+6 a_{2} x_{0}+\Phi}{2 \Phi} \operatorname{sn}^{2}\left(\frac{1}{2} \sqrt{\frac{\Phi}{3}}(t-T), \frac{\Phi-3 a_{1}-6 a_{2} x_{0}}{2 \Phi}\right)}{1+\frac{9 a_{1}+6 a_{2} x_{0}+\Phi}{2 \Phi} \operatorname{sn}^{2}\left(\frac{1}{2} \sqrt{\frac{\Phi}{3}}(t-T), \frac{\Phi-3 a_{1}-6 a_{2} x_{0}}{2 \Phi}\right)}\right]
$$

Then, the exact solution to Eq. (1) when $a_{1}>0$ and $a_{2}<0$ can be written as the following piecewise function

$$
x(t)=\left\{\begin{array}{cc}
x_{a}(t), & 0 \leq t \leq \frac{T}{4} \\
-x_{a}\left(t-\frac{T}{2}\right), & \frac{T}{4} \leq t \leq \frac{3 T}{4} \\
x_{a}(t-T), & \frac{3 T}{4} \leq t \leq T
\end{array}\right.
$$

where the exact period $T$ is given in Eq. (69). It is important to point out that when $a_{1}>0$ and $a_{2}<0$, Eqs. (59), (63), (64) and (65) are valid provided that the initial amplitude $x_{0}$ satisfies the condition $0<x_{0}<a_{1} /\left|a_{2}\right|$. 
In summary, in sections 2, 3 and 4 we obtained the exact periods and solutions for Eq. (1) for all values of parameters $a_{1}$ and $a_{2}$ and oscillation amplitude $x_{0}$ for which this system oscillates around the equilibrium position $x=0$. These values are shown in Eq. (48). In this case the period, $T$, and the periodic solution, $x$, are dependent on $x_{0}$ and $x \in\left[-x_{0}, x_{0}\right]$.

Figure 2 shows the variation in the period in Eq. (30) as a function of the initial amplitude $x_{0}$ when (a) $a_{1}=1$ and $a_{2}=1$, and (b) $a_{1}=-1$ and $a_{2}=1$, as well as the variation in the period in Eq. (59) when (c) $a_{1}=1$ and $a_{2}=-1$. Given that the exact solutions for motion are known, we can plot them as a function of time.

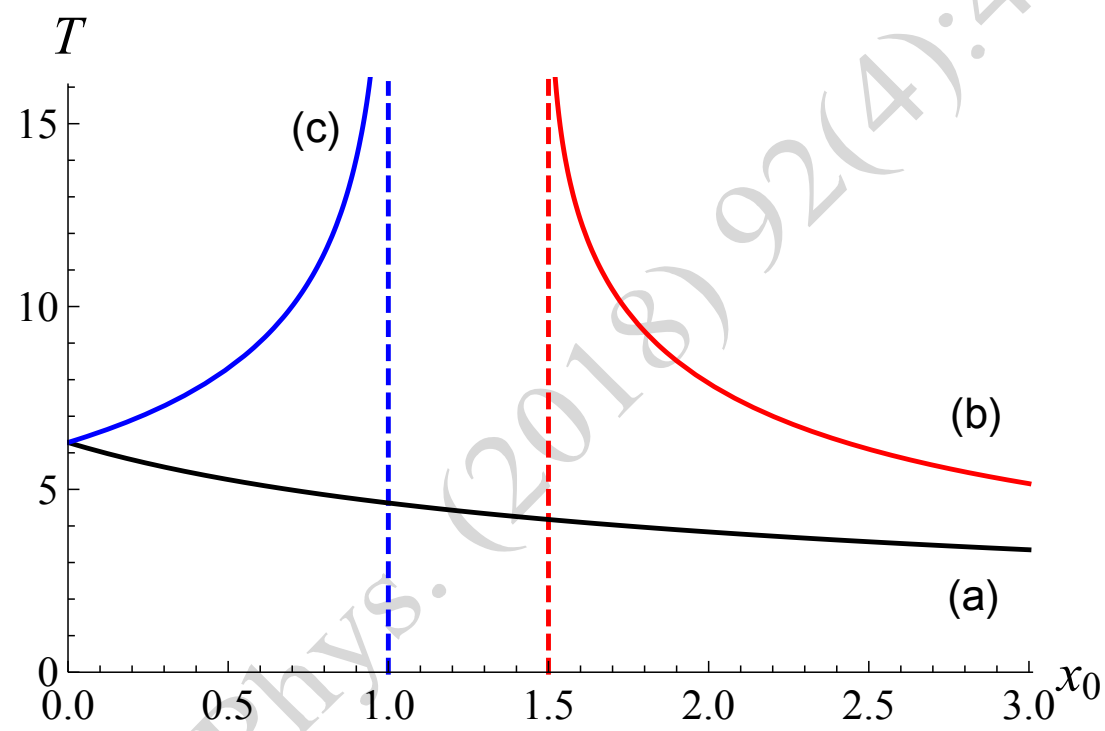

Figure 2.- Exact period in Eq. (30) as a function of the initial amplitude $x_{0}$ for (a) $a_{1}=1$ and $a_{2}=1$, and (b) $a_{1}=-1$ and $a_{2}=1$, as well as the variation in the period in Eq. (59) when (c) $a_{1}=$ 1 and $a_{2}=-1$.

Figure 3 shows the periodic solutions when (a) $a_{1}=1, a_{2}=1$ and $x_{0}=1$, and (b) $a_{1}=-1, a_{2}=$ 1 and $x_{0}=1.6$. These solutions were obtained using the period and exact solution given in Eqs. (27), (30), (40) and (43) and their phase plots are shown in Figures 1 (a) and (b), respectively. Finally, in Figure 3 (c) we plotted the exact solution when $a_{1}=1, a_{2}=-1$ and $x_{0}$ $=0.9$ using the period and exact solution given in Eqs. (59), (63), (64) and (65). The phase plot for this situation is shown in Figure 1 (c). 
(a)

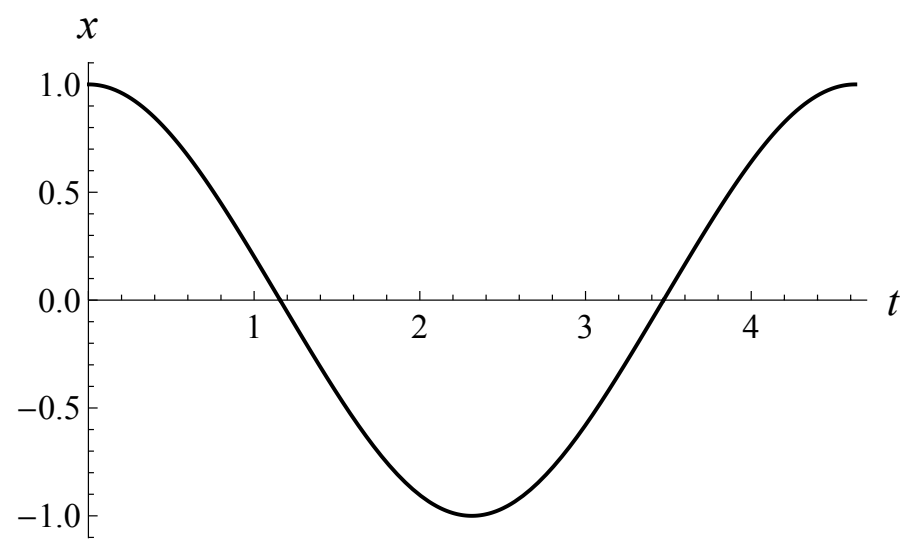

(b)

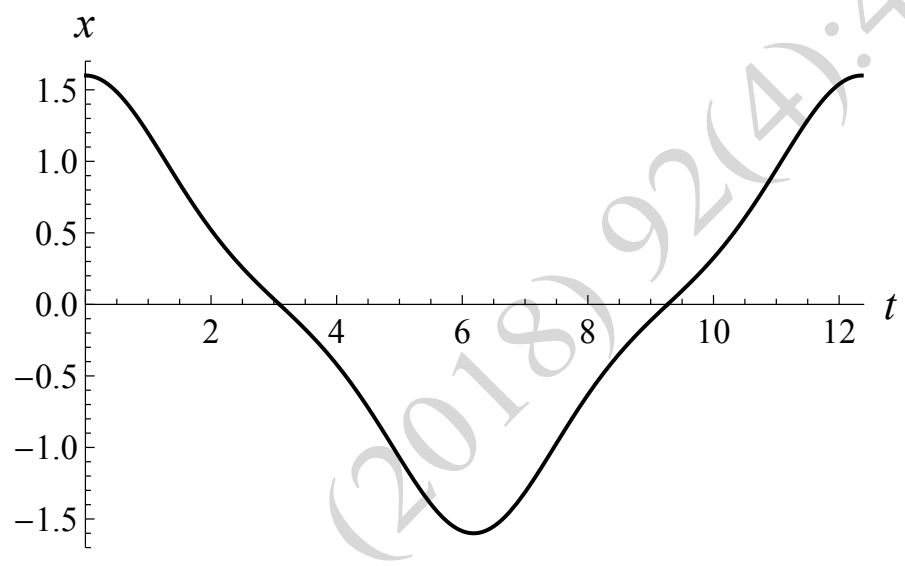

(c)

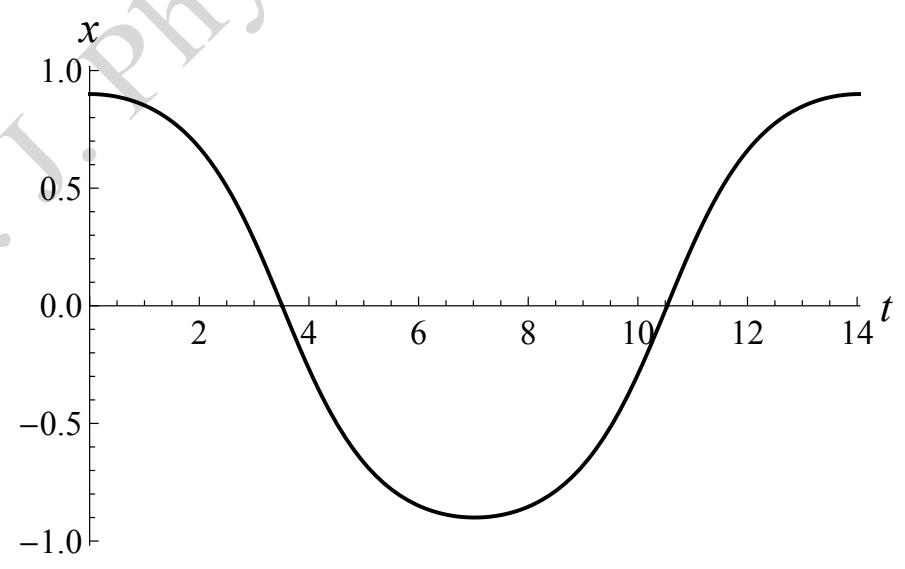

Figure 3.- Exact periodic solutions for an anti-symmetric quadratic oscillator when (a) $a_{1}=1, a_{2}=1$ and $x_{0}=1$ using the period and exact solution given in Eqs. (27), (30), (40) and (43), (b) $a_{1}=-1, a_{2}=1$ and $x_{0}=1.6$ using the period and exact solution given in Eqs. (27), (30), (40) and (43), and (c) $a_{1}=1, a_{2}=-1$ and $x_{0}=0.9$ using the exact period and solution given in Eqs. (59), (63), (64) and (65). 


\section{Solution when $a_{1}<0, a_{2}>0$ and $0<x_{0}<-3 a_{1} / 2 a_{2}$}

Now we assume that the coefficients of the linear and the nonlinear terms satisfy the condition

$$
a_{1}<0 \quad a_{2}>0
$$

with initial conditions

$$
x(0)=x_{0} \quad \frac{\mathrm{d} x}{\mathrm{~d} t}(0)=0
$$

Unlike in the cases studied above, the initial amplitude is now denoted by $x_{1}$. The phase plot in Figure $1(\mathrm{~b})$ shows the behaviour of the oscillator when $x_{0}>-\frac{3 a_{1}}{2 a_{2}}\left(a_{1}=-1, a_{2}=1\right.$ and $x_{0}$ $=8 / 5=1.6>3 / 2)$. The oscillation is periodic with a centre at $(0,0)$. In this situation, which was discussed in Section 3, Eqs. (27), (29), (40) and (43) must be used to obtain the exact period and solution replacing $A$ by $x_{1}$.

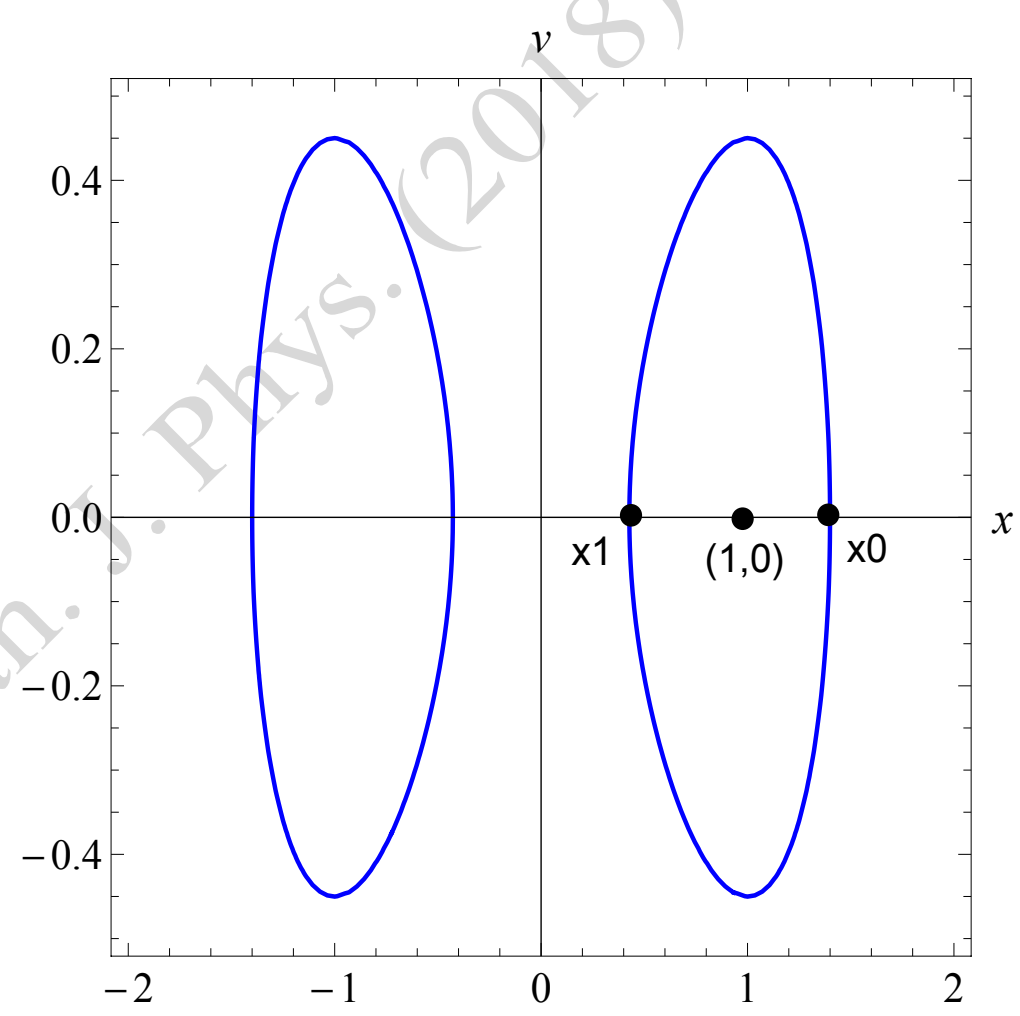

Figure 4.- Phase plot showing the behaviour of the oscillator when $0<x_{0}<-\frac{3 a_{1}}{2 a_{2}}\left(a_{1}=-1, a_{2}=1\right.$ and $x_{0}=7 / 5<3 / 2$ ). 
The phase portrait in Figure 4 shows the behaviour of the oscillator when $0<x_{0}<-\frac{3 a_{1}}{2 a_{2}}\left(a_{1}\right.$ $=-1, a_{2}=1$ and $\left.x_{0}=7 / 5=1.4<3 / 2\right)$. Three equilibrium points can be seen. There are centres at $\left( \pm a_{1} / a_{2}, 0\right)$ as well as a saddle at $(0,0)$. Now the system oscillates around the equilibrium position $x=-a_{1} / a_{2}$ (when $x \in\left[x_{1}, x_{0}\right]$ and $0<x_{1} \leq x \leq x_{0}$ ) or $x=a_{1} / a_{2}$ (when $\left.\frac{3 a_{1}}{2 a_{2}}<x_{0}<0\right)$. It is important to point out that now the differential equation given in Eq. (1) governs the motion of a mixed-parity nonlinear oscillator, $\frac{\mathrm{d}^{2} x}{\mathrm{~d} t^{2}}+a_{1} x+a_{2} x^{2}=0$, which has asymmetric quadratic nonlinearity with a positive quadratic term.

We shall now obtain the period and solution corresponding to the right orbit in Figure 4 for which $x>0$ considering that $x_{0}$ is the highest value of $x$. From Eq. (6) and considering that $x$ $>0$, we obtain $t$ as a function of $x$ as follows

$$
\sqrt{\frac{2 a_{2}}{3}} \mathrm{~d} t=-\frac{\mathrm{d} x}{\sqrt{\frac{3 a_{1}}{2 a_{2}}\left(x_{0}^{2}-x^{2}\right)+\left(x_{1}^{2}-x^{3}\right)}}
$$

which can be written as

$$
\sqrt{\frac{2 a_{2}}{3}} \int_{0}^{t} \mathrm{~d} t= \pm \int_{x_{0}}^{x} \frac{\mathrm{d} x}{\sqrt{\left(x_{0}-x\right)\left(x-x_{1}\right)\left(x-x_{2}\right)}}
$$

where $x_{1}$ and $x_{2}$ are defined by the following equations

$$
\begin{aligned}
& x_{1}=\frac{1}{4 a_{2}}\left(-3 a_{1}-2 a_{2} x_{0}+\sqrt{3} \sqrt{3 a_{0}^{2}-4 a_{1} a_{2} x_{0}-4 a_{2}^{2} x_{0}^{2}}\right) \\
& x_{2}=\frac{1}{4 a_{2}}\left(-3 a_{1}-2 a_{2} x_{0}-\sqrt{3} \sqrt{3 a_{1}^{2}-4 a_{1} a_{2} x_{0}-4 a_{2}^{2} x_{0}^{2}}\right)
\end{aligned}
$$

$x_{0}$ and $x_{1}$ are shown in Figure 4 when $a_{1}=-1, a_{2}=1$ and $x_{0}=7 / 5$. In this case the equilibrium point is at $x=-a_{1} / a_{2}(x=1$ and $v=0)$. It can easily be verified that the right orbit in Figure 4 satisfies the inequalities

$$
x_{0} \geq x \geq x_{1}>x_{2}
$$


Now the period, $T$, and the periodic solution, $x$, are dependent on $x_{0}$ and $x \in\left[x_{1}, x_{0}\right]$. The following integral is valid for $a \geq x \geq b>c\left[17,6^{\text {th }}\right.$ edition, Formula 3.131 , Integral 6 , page 250]

$$
\int_{x}^{a} \frac{\mathrm{d} x}{\sqrt{(a-x)(x-b)(x-c)}}=\frac{2}{\sqrt{a-c}} F(\lambda, p)
$$

where $F(\lambda, p)$ is the incomplete elliptic integral of the first kind defined in Eq. (15) and $\lambda$ and $p$ are given by the following equations

$$
\begin{gathered}
\lambda=\arcsin \sqrt{\frac{a-x}{a-b}} \\
p=\frac{a-b}{a-c}
\end{gathered}
$$

Then the value of the integral in Eq. (70) is

$$
t=2 \sqrt{\frac{3}{2 a_{2}\left(x_{0}-x_{2}\right)}} F\left(\arcsin \sqrt{\frac{x_{0}-x}{x_{0}-x_{1}}}, \frac{x_{0}-x_{1}}{x_{0}-x_{2}}\right)
$$

As can be seen in Figure 4, the period of oscillation is twice the time taken by the oscillator to go from $x=x_{0}$ to $x=x_{1}$. Therefore

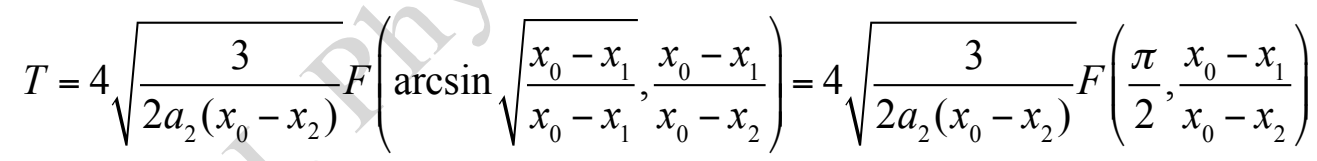

which finally can be written as follows

$$
T=4 \sqrt{\frac{3}{2 a_{2}\left(x_{0}-x_{2}\right)}} K\left(\frac{x_{0}-x_{1}}{x_{0}-x_{2}}\right)
$$

where $K(m)$ is the complete elliptic integral of the first kind defined as follows [16]

$$
K(m)=\int_{0}^{\pi / 2} \frac{\mathrm{d} \theta}{\sqrt{1-m \sin ^{2} \theta}}
$$

Substituting Eqs. (71) and (72) in Eq. (79), gives the exact period of oscillation as 


$$
T=\frac{4 \sqrt{6}}{\sqrt{3 a_{1}+6 a_{2} x_{0}+\sqrt{3} \sqrt{3 a_{1}^{2}-4 a_{1} a_{2} x_{0}-4 a_{2}^{2} x_{0}^{2}}}} K\left(\frac{3 a_{1}+6 a_{2} x_{0}-\sqrt{3} \sqrt{3 a_{1}^{2}-4 a_{1} a_{2} x_{0}-4 a_{2}^{2} x_{0}^{2}}}{3 a_{1}+6 a_{2} x_{0}+\sqrt{3} \sqrt{3 a_{1}^{2}-4 a_{1} a_{2} x_{0}-4 a_{2}^{2} x_{0}^{2}}}\right)
$$

which satisfies the condition $T\left(x_{1}\right)=T\left(x_{0}\right)$. Taking into account the definition given in Eq. (60), the period can also be written as follows

$$
T=\frac{4 \sqrt{6}}{\sqrt{3 a_{1}+6 a_{2} x_{0}+\Phi}} K\left(\frac{3 a_{1}+6 a_{2} x_{0}-\Phi}{3 a_{1}+6 a_{2} x_{0}+\Phi}\right)
$$

In order to obtain the solution $x$ as a function of time $t$, from Eq. (77) it follows that

$$
F\left(\arcsin \sqrt{\frac{x_{0}-x}{x_{0}-x_{1}}}, \frac{x_{0}-x_{1}}{x_{0}-x_{2}}\right)=\frac{1}{2} \sqrt{\frac{2 a_{2}}{3}}\left(x_{0}-x_{2}\right)^{1 / 2} t
$$

which may be written as

$$
\sqrt{\frac{x_{0}-x}{x_{0}-x_{1}}}=\operatorname{sn}\left(\frac{1}{2} \sqrt{\frac{2 a_{2}}{3}}\left(x_{0}-x_{2}\right)^{1 / 2} t, \frac{x_{0}-x_{1}}{x_{0}-x_{2}}\right)
$$

Then the exact solution is given by the equation

$$
x(t)=x_{0}-\left(x_{0}-x_{1}\right) \operatorname{sn}^{2}\left(\frac{1}{2} \sqrt{\frac{2 a_{2}}{3}}\left(x_{0}-x_{2}\right)^{1 / 2} t, \frac{x_{0}-x_{1}}{x_{0}-x_{2}}\right)
$$

Finally, taking into account Eqs. (60), (71) and (72) the exact solution may be expressed as

$$
x(t)=x_{1}-\frac{1}{4 a_{2}}\left(3 a_{1}+6 a_{2} x_{0}-\Phi\right) \operatorname{sn}^{2}\left(\frac{\sqrt{3 a_{1}+6 a_{2} x_{0}+\Phi}}{2 \sqrt{6}} t, \frac{3 a_{1}+6 a_{2} x_{0}-\Phi}{3 a_{1}+6 a_{2} x_{0}+\Phi}\right)
$$

When $a_{1}<0$ and $a_{2}>0$, Eqs. (82) and (86) are valid provided that the initial position $x_{0}$ satisfies the condition $0<x_{0}<\frac{3 a_{1}}{2\left|a_{2}\right|}$, except for the equilibrium point (when $x_{0}=a_{1} /\left|a_{2}\right|$ ). As can be seen, in this case the exact solution is not a piece-wise function and using only one equation it is possible to describe the oscillatory motion of the system for any value of time. 
The expressions for the period and exact solution corresponding to the left orbit in Figure 4, i.e., when $x<0$ can be obtained following the same procedure as that used when $x>0$ and is not included here.

Figure 5 shows the variation in period as a function of initial position when $a_{1}=-1$ and $a_{2}=$ 1. (a) $0<x_{0}<-3 a_{1} / 2 a_{2}=3 / 2$, section 5, Eq. (81), and (b) $x_{0}>-3 a_{1} / 2 a_{2}=3 / 2$, section 3, Eq. (30). As can be seen the motion is not periodic when the initial position is $-3 a_{1} / 2 a_{2}$. Figure 6 (a) shows the plot of displacement $x$ as a function of time $t$ when $a_{1}=-1, a_{2}=1$ and $x_{0}=7 / 5$ $=1.4$. The displacement was obtained using Eq. (86). In this example, and from Eq. (81), it follows that

$$
x_{1}=\frac{1}{2}(1+\sqrt{57}) \approx 0.427492
$$

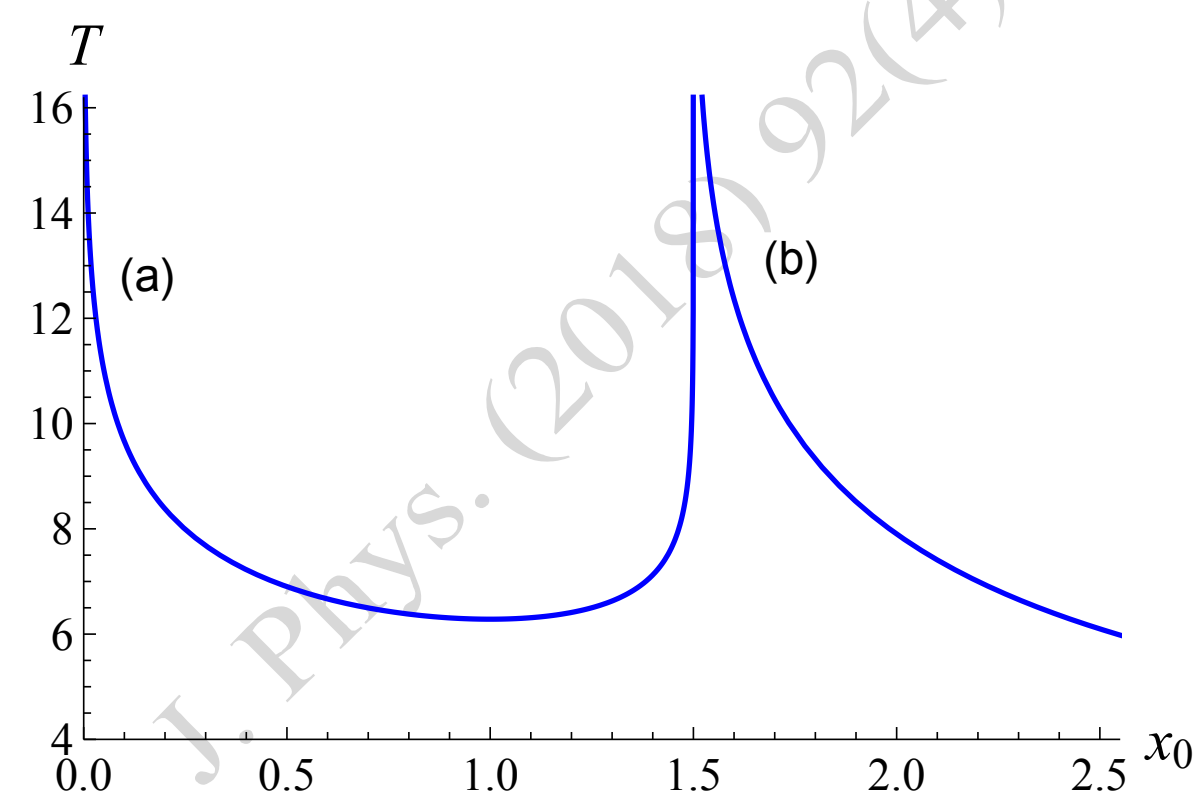

Figure 5.- Exact period as a function of the initial position $x_{0}$ for $a_{1}=-1$ and $a_{2}=1$. (a) $0<x_{0}<-\frac{3 a_{1}}{2 a_{2}}=\frac{3}{2}$, section 5, Eq. (81), and (b) $x_{0}>-\frac{3 a_{1}}{2 a_{2}}=\frac{3}{2}$, section 3, Eq. (30).

As can be easily verified in Figure 6 (a), the oscillatory motion of this system is bounded between $x_{1}=\frac{1}{2}(1+\sqrt{57}) \approx 0.427492$ and $x_{0}=7 / 5=1.4$ and the equilibrium point is located at $x=a_{1} /\left|a_{2}\right|=1$. 
(a)

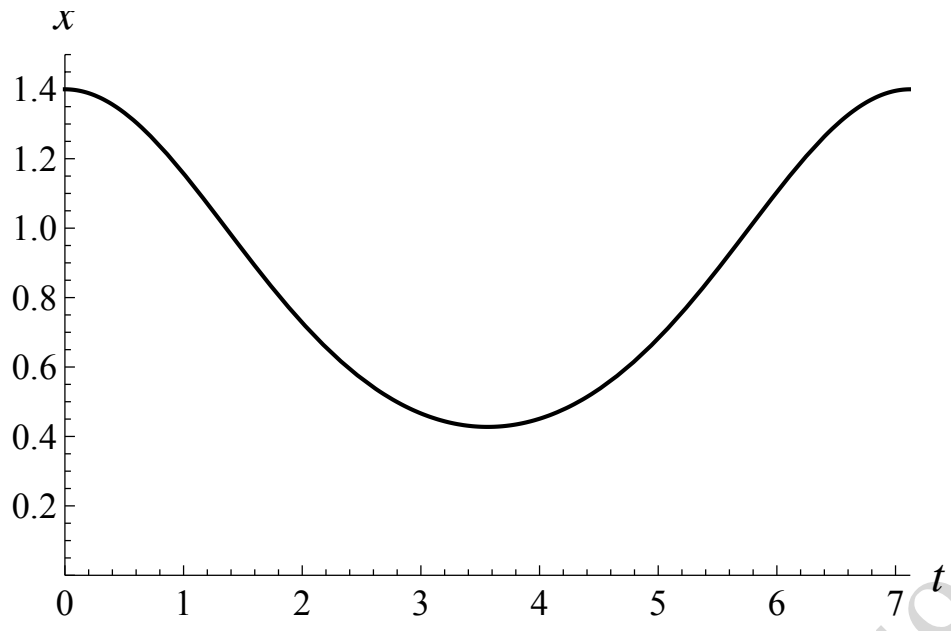

(b)

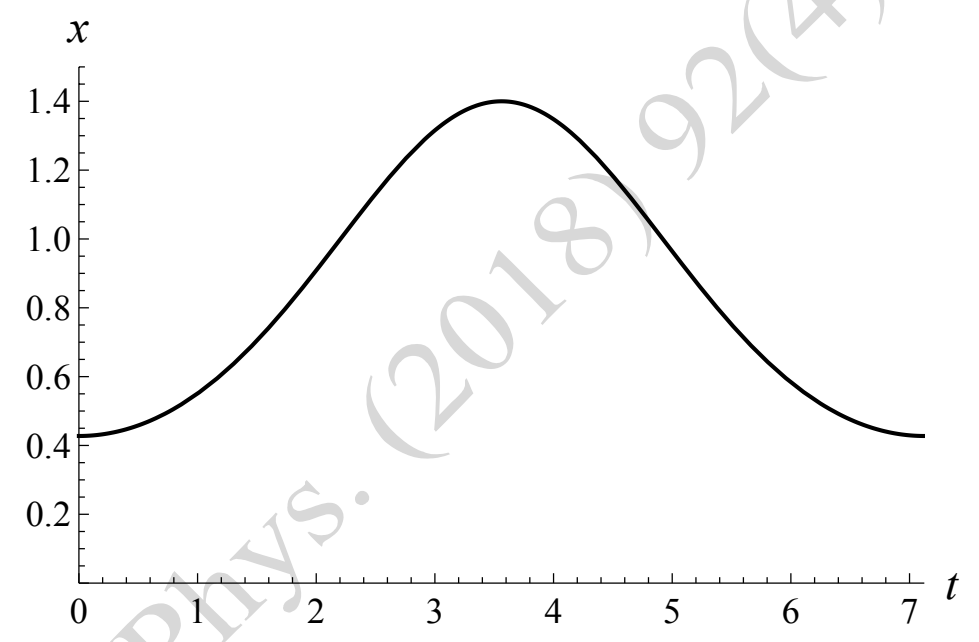

Figure 6.- Exact periodic solution for an anti-symmetric quadratic oscillator when (a) $a_{1}=-1, a_{2}=1$ and $x_{0}=7 / 5=1.4$. This displacement was obtained using Eq. (86), and (b) $a_{1}=-1, a_{2}=1$ and $x_{0}=\frac{1}{2}(1+\sqrt{57}) \approx 0.427492$. In this situation, $x_{1}=7 / 5=1.4$.

Figure 6 (b) shows displacement $x$ as a function of time $t$ when $a_{1}=-1, a_{2}=1$ and $x_{0}=\frac{1}{2}(1+\sqrt{57}) \approx 0.427492$. In this case $x_{1}=7 / 5=1.4$. The equation of the separatrix orbit may be obtained from Eq. (86). For this orbit $x_{0}=-3 a_{1} / 2 a_{2}$ and the period $T$ in Eq. (82) is infinite. Substituting $x_{0}=-3 a_{1} / 2 a_{2}$ in Eq. (86) gives 
Eq. (86) allows us to obtain the equation of the separatrix orbit. For this orbit $x_{0}=-3 a_{1} / 2 a_{2}$ and the period $T$ in Eq. (82) is infinite. Substituting $x_{0}=-3 a_{1} / 2 a_{2}$ in Eq. (86) we obtain

$$
x_{a s}(t)=-\frac{3 a_{1}}{2 a_{2}}+\frac{3 a_{1}}{2 a_{2}} \tanh ^{2}\left(\frac{1}{2} \sqrt{-a_{1}} t\right)
$$

and the velocity is

$$
v_{a s}(t)=\frac{3 a_{1} \sqrt{-a_{1}}}{2 a_{2}} \tanh \left(\frac{1}{2} \sqrt{-a_{1}} t\right)\left[1-\tanh ^{2}\left(\frac{1}{2} \sqrt{-a_{1}} t\right)\right]
$$

which gives the following relationship between $v_{a s}$ and $x_{a s}$

$$
v_{a s}^{2}\left(x_{a s}\right)=-\frac{1}{3} x_{a s}^{2}\left(3 a_{1}+2 a_{2} x_{a s}\right)
$$

for $x_{0}=-3 a_{1} / 2 a_{2}>0$ and

$$
v_{a s}^{2}\left(x_{a s}\right)=-\frac{1}{3} x_{a s}^{2}\left(3 a_{1} \pm 2 a_{2} x_{a s}\right)
$$

for $x_{0}=3 a_{1} / 2 a_{2}<0$. For both initial conditions, $x_{0}=-3 a_{1} / 2 a_{2}>0$ and $x_{0}=3 a_{1} / 2 a_{2}<0$, the separatrix curve has a fish-shaped form and corresponds to a homoclinic orbit connecting the hyperbolic fixed point $x=0$ to itself. Figure 7 shows the phase-space curves when $a_{1}=-$ 1 and $a_{2}=1$ and combines the results analysed in sections 3 and 5. The separatrix curve calculated using Eq. (91) is also plotted in this figure. There are three critical points, $\left(a_{1} / a_{2}, 0\right)$ and $\left(-a_{1} / a_{2}, 0\right)$, which are centres, and $(0,0)$, which is an unstable saddle point.

\section{Conclusions}

Oscillators with symmetric quadratic nonlinearity were analysed and closed-form expressions for their exact periods and solutions for all possible oscillatory motions were obtained. Four possible combinations of coefficients $a_{1}$ and $a_{2}$ were shown to give periodic motions. In three of them ((a) $a_{1} \geq 0, a_{2}>0$, and $x_{0}>0$, (b) $a_{1}<0, a_{2}>0$ and $x_{0}>-3 a_{1} / 2 a_{2}$, and (c) $a_{1}>0, a_{2}<0$ and $0<x_{0}<-a_{1} / a_{2}$ ) the system oscillates around the equilibrium position $x=0$ with $x \in\left[-x_{0}, x_{0}\right]$, where $x_{0}>0$ is the oscillation amplitude. However, there is still one more case ((d) $a_{1}>0, a_{2}<0$, and $0>x_{0}>-3 a_{1} / 2 a_{2}$ or $\left.3 a_{1} / 2 a_{2}<x_{0}<0\right)$ in which the system does not oscillate around the position $x=0$ with $x \in\left[-x_{0}, x_{0}\right]$ but around the 
equilibrium position $x=-a_{1} / a_{2}$ with $x \in\left[x_{1}, x_{0}\right]$ (when $0>x_{0}>-3 a_{1} / 2 a_{2}$ ) or $x=a_{1} / a_{2}$ with $x \in\left[x_{1}, x_{0}\right]$ (when $3 a_{1} / 2 a_{2}<x_{0}<0$ ). Case (d) corresponds to a mixed-parity nonlinear oscillator with asymmetric quadratic nonlinearity and a positive quadratic term.

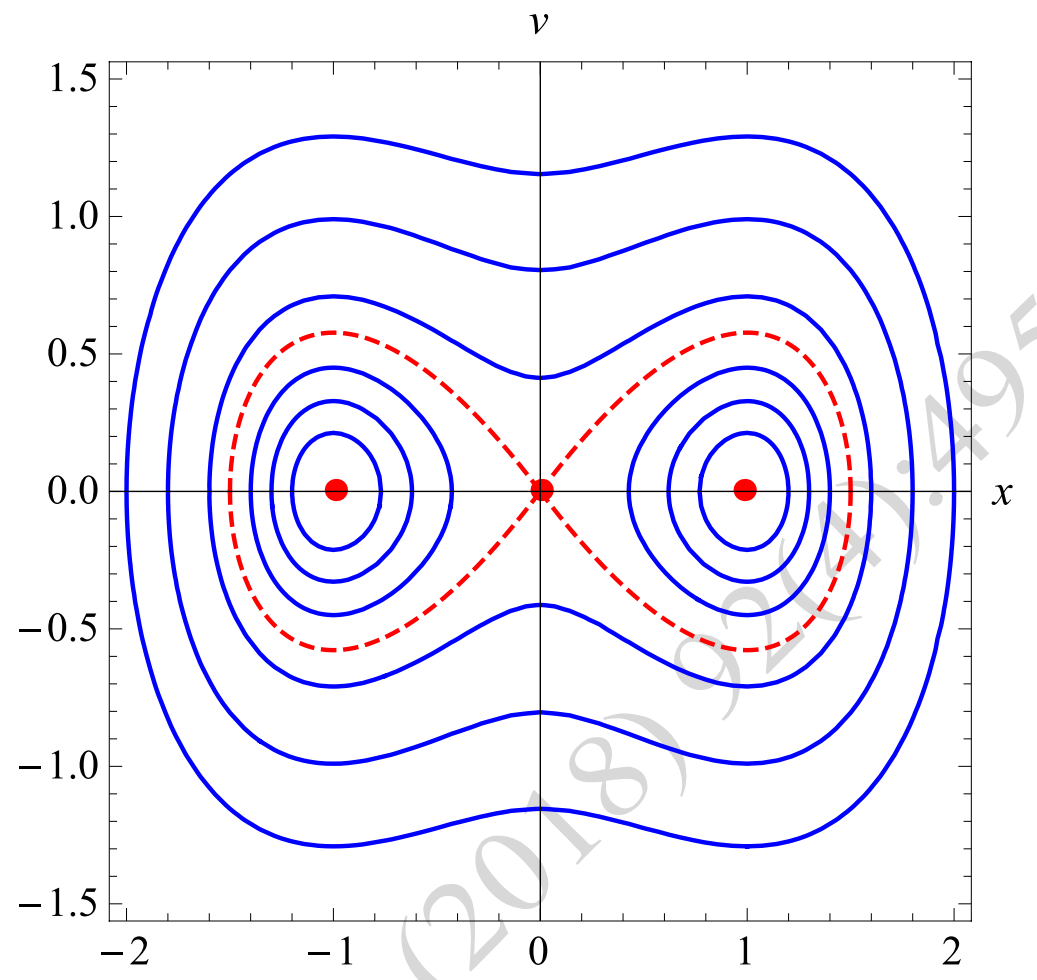

Figure 7.- Phase-space curves when $a_{1}=-1$ and $a_{2}=1$ combining the results analysed in sections 3 and 5. The separatrix orbit (dashed line) as well as the critical points $\left( \pm a_{1} / a_{2}, 0\right)$, which are centres, and $(0,0)$, which is an unstable saddle point, are also included.

In cases (a) and (b) the exact period is given as a function which includes an incomplete elliptic integral of the first kind and the exact solution is expressed as a piecewise function including Jacobi elliptic cosine functions. In case (c) the exact period also includes an incomplete elliptic integral of the first kind and the exact solution is also expressed as a piecewise function but now it includes Jacobi elliptic sine functions. Finally, in case (d) the exact period is given as a function which includes an incomplete elliptic integral of the first kind, whereas the exact solution consists of a single function including Jacobi elliptic sine functions. It was necessary to solve an integral (Eq. (9)) in order to obtain the exact solution in cases (a) and (b). We found an error in the calculation of this integral in GR's book [17] and so we solved it ourselves. 


\section{Appendix A}

In GR's book [17, $6^{\text {th }}$ edition, Formula 3.138, Integral 8, page 260] the solution of the following integral may be found

$$
I=\int_{x}^{\alpha} \frac{\mathrm{d} x}{\sqrt{(\alpha-x)\left[(x-\sigma)^{2}+\rho^{2}\right]}}
$$

This is the integral we needed to solve in Eq. (9) in order to obtain the exact period of the nonlinear oscillator modelled by Eq. (1). The solution given in GR's book is

$$
I=\int_{x}^{\alpha} \frac{\mathrm{d} x}{\sqrt{(\alpha-x)\left[(x-\sigma)^{2}+\rho^{2}\right]}}=\frac{1}{\sqrt{p}} F\left(2 \operatorname{arccot} \sqrt{\frac{\alpha-x}{p}}, \frac{p-\sigma+\alpha}{2 p}\right)
$$

where

$$
p=\sqrt{(\sigma-\alpha)^{2}+\rho^{2}}
$$

and $x<\alpha$. However, this solution is incorrect as shown below.

We consider the integral in Eq. (A1). The change of variable

$$
\tan \frac{\theta}{2}=\sqrt{\frac{\alpha-x}{p}}
$$

gives

$$
I=\int_{0}^{2 \arctan \sqrt{\frac{\alpha-x}{p}}} \frac{p \tan (\theta / 2) \mathrm{d} \theta}{\cos ^{2}(\theta / 2) \sqrt{p \tan ^{2}(\theta / 2)\left[\left(\alpha-\sigma-p \tan ^{2}(\theta / 2)\right)^{2}+\rho^{2}\right]}}
$$

which after some mathematical operations can be written as follows

$$
I=\frac{1}{\sqrt{p}} \int_{0}^{2 \arctan \sqrt{\frac{\alpha-x}{p}}} \frac{\mathrm{d} \theta}{\sqrt{\cos ^{4}(\theta / 2)+\sin ^{4}(\theta / 2)+\frac{2(\sigma-\alpha)}{p} \sin ^{2}(\theta / 2) \cos ^{2}(\theta / 2)}}
$$

Taking into account the following trigonometric equations

$$
\cos ^{4} \frac{\theta}{2}+\sin ^{4} \frac{\theta}{2}=\left(\frac{1+\cos \theta}{2}\right)^{2}+\left(\frac{1-\cos \theta}{2}\right)^{2}=\frac{1}{2}\left(1+\cos ^{2} \theta\right)=1-\frac{1}{2} \sin ^{2} \theta
$$




$$
\sin ^{2} \frac{\theta}{2} \cos ^{2} \frac{\theta}{2}=\frac{1}{4} \sin ^{2} \theta
$$

Eq. (A5) becomes

$$
I=\frac{1}{\sqrt{p}} \int_{0}^{2 \arctan \sqrt{\frac{\alpha-x}{p}}} \frac{\mathrm{d} \theta}{\sqrt{1-\frac{1}{2} \sin ^{2} \theta+\frac{\sigma-\alpha}{2 p} \sin ^{2} \theta}}=\frac{1}{\sqrt{p}} \int_{0}^{2 \arctan \sqrt{\frac{\alpha-x}{p}}} \frac{\mathrm{d} \theta}{\sqrt{1-\frac{p-\sigma+\alpha}{2 p} \sin ^{2} \theta}}
$$

Now considering an incomplete elliptic integral of the first kind defined in Eq. (15) and introducing $\phi$ and $m$ as follows

$$
\begin{gathered}
\phi=2 \arctan \sqrt{\frac{\alpha-x}{p}} \\
m=\frac{p-\sigma+\alpha}{2 p}
\end{gathered}
$$

the correct solution for the integral in Eq. (A1) is

$$
I=\int_{x}^{\alpha} \frac{\mathrm{d} x}{\sqrt{(\alpha-x)\left[(x-\sigma)^{2}+\rho^{2}\right]}}=\frac{1}{\sqrt{p}} F\left(2 \arctan \sqrt{\frac{\alpha-x}{p}}, \frac{p-\sigma+\alpha}{2 p}\right)
$$

which is the equation we used in section 2 .

\section{Acknowledgements}

This work was supported by the "Generalitat Valenciana" of Spain, under project PROMETEOII/2015/015 and by the "Vicerrectorado de Tecnologías de la Información" of the University of Alicante, Spain, under project GITE-09006-UA. 
A. Beléndez et al. Indian Journal of Physics - ISSN 0973-1458

Indian J Phys (2018) 92(4):495-506 DOI 10.1007/s12648-017-1125-9

\section{References}

[1] A H Nayfeh Problems in Perturbation (New York : John Wiley \& Sons, 1985)

[2] R E Mickens Oscillations in Planar Dynamics Systems (Singapore : World Scientific, 1996)

[3] A H Nayfeh and D T Mook Nonlinear Oscillations (New York : Wiley-Interscience, 1979)

[4] L Cveticanin Strongly Nonlinear Oscillators: Analytical Solutions (Heidelberg : Springer, 2014)

[5] R E Mickens and I Ramadhani J. Sound Vib. 155190 (1992)

http://dx.doi.org/10.1016/0022-460X(92)90657-J

[6] L Cveticanin Physica A 341, 123 (2004)

http://dx.doi.org/10.1016/j.physa.2004.04.123

[8] A Elías-Zúniga Appl. Math. Comput. 218, 7590 (2012)

http://dx.doi.org/10.1016/j.amc.2012.01.025

[7] J-W Zhu Appl. Math. Modell. 38, 5986 (2014)

http://dx.doi.org/10.1016/j.apm.2014.04.065

[9] V Marinca and N Herişanu Math. Comput. Modell. 53, 604 (2011)

http://doi.org/10.1016/j.mcm.2010.09.011

[10] R E Mickens Truly Nonlinear Oscillations (Singapore : World Scientific, 2010)

[11] A Beléndez, E Arribas, C Pascual, T Beléndez, M L Alvarez and A Hernández Appl. Math. Comput. 246, 355 (2014)

http://dx.doi.org/10.1016/j.amc.2014.08.005 
A. Beléndez et al. Indian Journal of Physics - ISSN 0973-1458

Indian J Phys (2018) 92(4):495-506 DOI 10.1007/s12648-017-1125-9

[12] R E Mickens and M Mixon J. Sound Vib. 159, 546 (1992).

http://dx.doi.org/10.1016/0022-460X(92)90759-Q

[13] S H Chen, X M Yang and Y K Cheung J. Sound Vib. 212, 771 (1998).

http://dx.doi.org/10.1006/jsvi.1997.1411

[14] A Beléndez, C Pascual, T Beléndez and A Hernández Nonlinear Analysis: Real World Applications 10, 416 (2009)

http://dx.doi.org/10.1016/j.nonrwa.2007.10.002

[15] A Beléndez, E Gimeno, M L Alvarez, M S Yebra and D I Méndez Int. J. Comput. Math. 87, 1497 (2010)

http://dx.doi.org/10.1080/00207160802380942

[16] L Cveticanin, M Zukovic, Gy Mester, I Biro and J Sarosi Acta Mechanica 227, 1727 (2016) http://dx.doi.org/10.1007/s00707-016-1582-9

[17] I S Gradshteyn and I M Ryzhik Table of Integrals, Series and Products. Sixth Edition. (San Diego : Academic Press, 2000)

[18] L M Milne-Thomson "Elliptic integrals" Ch. 17 in Handbook of Mathematical functions with Formulas, Graphics and Mathematical Tables. M. Abramowitz and I. A. Stegun (Eds.). New York, Dover, pp. 587-607, 1972.

[19] L M Milne-Thomson, "Jacobian elliptic functions and theta functions" Ch. 16 in Handbook of Mathematical functions with Formulas, Graphics and Mathematical Tables. M. Abramowitz and I. A. Stegun (Eds.). New York, Dover, pp. 567-581, 1972. 